\title{
BMJ Open Digital Dementia Registry Bavaria- digiDEM Bayern: study protocol for a multicentre, prospective, longitudinal register study
}

Nikolas Dietzel (D , ${ }^{1}$ Lara Kürten, ${ }^{1}$ Linda Karrer, ${ }^{1}$ Michael Reichold, ${ }^{2}$ Laura Köhler, ${ }^{3}$ Andreas Nagel, ${ }^{1}$ Christina Chmelirsch, ${ }^{1}$ Kathrin Seebahn, ${ }^{1}$ Markus Hladik, ${ }^{1}$ Sebastian Meuer, ${ }^{1}$ Anna Kirchner, ${ }^{1}$ Kristina Holm, ${ }^{1}$ Marina Selau, ${ }^{2}$ Marco Wendel, ${ }^{3}$ Jörg Trinkwalter, ${ }^{3}$ Hans-Ulrich Prokosch, ${ }^{2}$ Elmar Graessel, ${ }^{4}$

Peter L Kolominsky-Rabas (1) ${ }^{1}$

To cite: Dietzel N, Kürten L, Karrer L, et al. Digital Dementia Registry BavariadigiDEM Bayern: study protocol for a multicentre, prospective, longitudinal register study. BMJ Open 2021;11:e043473. doi:10.1136/ bmjopen-2020-043473

- Prepublication history for this paper is available online. To view these files, please visit the journal online (http://dx.doi org/10.1136/bmjopen-2020043473).

Received 06 August 2020 Revised 25 November 2020 Accepted 27 November 2020

Check for updates

(C) Author(s) (or their employer(s)) 2021. Re-use permitted under CC BY-NC. No commercial re-use. See rights and permissions. Published by BMJ.

For numbered affiliations see end of article.

Correspondence to

Mr Nikolas Dietzel;

nikolas.dietzel@fau.de

\section{ABSTRACT}

Introduction Dementia is one of the most relevant widespread diseases, with a prevalence of currently 50 million people with dementia worldwide. The care of people with dementia will be one of the major challenges for healthcare systems worldwide. Digitalisation offers new possibilities to improve both dementia healthcare and health outcomes research as a fundament for national healthcare planning. The 'Digital Dementia Registry Bavaria-digiDEM Bayern' aims to improve the understanding of the complexity and long-term progression of dementia and the current care situation in Bavaria. Moreover, by offering digital services, digiDEM will actively contribute to improving the care situation in Bavaria. Methods and analysis digiDEM will recruit people with dementia and their family caregivers in all administrative regions of Bavaria. All participants will undergo dementia screening prior to study inclusion in order to identify people with mild cognitive impairment and mild-to-moderate dementia. Participants will be followed up over a period of three years. Sociodemographic data, type of dementia, symptoms, diagnosis, cognitive trajectories, activities of daily living, behavioural and psychological symptoms, falls, resource utilisation, caregiver burden, quality of life, needs of people with dementia and their caregivers, mobility, use of media and sources of information will be assessed. The project will implement a digital web-based platform for data collection. Data will be collected by means of standardised online or face-to-face interviews.

Ethics and dissemination The study obtained ethical approval from the Ethics Committee of the Medical Faculty of Friedrich-Alexander-University Erlangen-Nürnberg (FAU) (application number: 253_20 B). Findings will be used for evidence-based decision-making for health decisionmakers in order to optimise dementia healthcare in the state of Bavaria. Specific analyses will be conducted for the participating research partners. Results of the study will be published in peer-reviewed journals.

\section{INTRODUCTION}

Digitalisation and technology show the potential to change healthcare

\section{Strengths and limitations of this study}

- The 'Digital Dementia Registry Bavaria-digiDEM Bayern' will provide health outcomes data necessary for healthcare planning while simultaneously improving the care situation by offering digital services.

- The registry will include people with dementia from a broad range of recruiting facilities without restrictions of age or type of dementia (excluding people in severe states of dementia) and with a focus on often-neglected rural areas.

- The project will use a web-based data entry system for data collection, improving data quality by facilitating plausibility tests in order to minimise the danger of missing or erroneous data during data collection and to reduce errors during the data transmission process.

- There is no random sampling, so a selection bias of people with specific care backgrounds cannot be ruled out.

fundamentally. ${ }^{12}$ Therefore, the digital transformation of healthcare systems is becoming a highly political issue. In 2019, the WHO published a guideline including recommendations on digital interventions for health system strengthening, following a WHO resolution on digital health of $2018 .^{3}$ The WHO considers digital technologies as a crucial tool 'to tackle health system challenges, and thereby offer the potential to enhance the coverage and quality of health practices and services' and therefore to achieve universal health coverage. ${ }^{3}$ The European Commission proclaims that 'digital solutions for health and care can increase the well-being of millions of citizens and radically change the way health and care services are delivered to 
patients'. ${ }^{4}$ The three central aims the Commission wants to achieve are (1) citizens' secure access to and sharing of health data across borders, (2) better data to advance research, disease prevention, and personalised health and care, and (3) digital tools for citizen empowerment and person-centred care. ${ }^{45}$

Dementia is one of the main public health challenges of current and future societies worldwide. Recently, the WHO has declared dementia as a public health priority. ${ }^{6}$ With currently about 50 million affected people, dementia is one of the diseases with the highest prevalence worldwide. Due to the demographic change, the global prevalence is expected to increase to more than 152 million by $2050 .{ }^{7}$ The growing prevalence is accompanied by a tremendous cost increase, as dementia is already one of the most cost-intensive diseases of older adults. ${ }^{8}$ The main cost drivers in a community-based setting are informal care costs $(60 \%-84 \%$ of the total costs) due to home-based long-term care and nursing home expenditures rather than direct medical costs (inpatient and outpatient services as well as medication). ${ }^{9}$ The extent of further cost components differs between countries. In the UK and France, patient social care costs are the second-highest cost component, whereas, in Germany, it is patient healthcare cost. All cost components increase over the course of the disease. ${ }^{10}$

\section{Challenges in dementia care}

International research illustrates several challenges in dementia healthcare, which are the fundament for setting up the project 'Digital Dementia Registry Bavaria-digiDEM Bayern'. These findings were also proved in the predecessor project 'Bavarian Dementia Registry-BayDem'. ${ }^{11}$

One major finding is the long duration between the perception of the first symptoms and the diagnosis. Studies showed an average time from 1.3 to 3.1 years between the appearance of the first symptoms and diagnosis. ${ }^{11-14}$ In this respect, Barth $e t a l^{15}$ showed that access to medical examinations and timely diagnoses are central problems in rural areas. Digital solutions, however, have the potential to support dementia diagnosis in rural regions. A further issue is the situation of family caregivers, as most of the people with dementia are cared for at home by a relative. ${ }^{6}{ }^{16}$ Caregivers, which are predominately women, often experience a high caregiver burden that can lead to higher morbidity and mortality. ${ }^{17-19}$ Thus, caregivers of people with dementia are often referred to as the invisible second patient. ${ }^{20}$ Caregiver burden can be effectively reduced by using care services. The importance of those services increases alongside the care dependencies of the people with dementia over the course of the disease. However, international literature shows low utilisation rates of care services despite a broad range of available services. ${ }^{1121-23}$ Differences between people living in urban and people living in rural areas are reflected in the utilisation of these services, where the use of specific care services is lower in rural areas. ${ }^{11}$ There are country and state-specific differences on which type of service has low utilisation rates. In Bavaria, utilisation of supervision groups, transport service and 'meals on wheels' was below $10 \% .{ }^{24}$ Other studies report low utilisation rates, in particular of respite care ${ }^{23}$ or day care. ${ }^{25}$ Those challenges are intensified, as information about the diagnosis, the course of the disease, as well as pharmacological and psychosocial treatments are often insufficient, in particular in rural areas. ${ }^{11} 26$

\section{Potential of digital technologies in dementia healthcare and research}

In order to tackle the current and upcoming challenges of dementia healthcare, digitalisation has the potential to offer useful tools and solution approaches. In this regard, digital technologies can be used in multiple ways along with the entire disease and care process. A central benefit of digital technologies, such as digital platforms, can be the provision of broad access for health-related information and interventions for citizens, regardless of the location. ${ }^{3627}$ Thus, digital technologies can contribute to empowering citizens to initiate diagnosis and treatment processes and therefore fostering the process of shared decision-making. ${ }^{327} 28$ That is particularly important for people living in rural areas, characterised by a low density of health services, like counselling or diagnosis. ${ }^{15} 2629$ The low-threshold online access to information about dementia in general and its different subtypes, their diagnosis and treatment, and helpful (medical) contacts can strengthen dementia management for people in rural areas. ${ }^{1526} 27$ Moreover, online information is particularly relevant for dementia as a disease that causes stigmas by supporting the confrontation with the disease and encouraging seeking support without fearing stigmatisation. In addition, affected people can get in touch with each other. ${ }^{30}$ At the same time, online information can contribute to diminishing stigmas by bringing people in touch with dementia. ${ }^{27}$ The more people gain knowledge and understanding of dementia, the better they are able to identify dementia-caused behaviour and to help and include people with dementia in societal life. In this regard, an advantage of online content is that a broad amount of information can be provided in one single place (one-stop-shop) and be frequently updated. Simultaneously, digital platforms can provide orientation in understanding and structuring this information. ${ }^{27}$

However, digital technologies are not solely useful for the provision of information but also to enable citizens to take an active role in their own dementia management, for example, by monitoring the course of the disease, providing online treatment and training measures, or giving frequent reminders for health management activities, like medication adherence. ${ }^{31}$ The tracking of health-related information can furthermore support the decision-making and treatment process in collaboration with health workers. ${ }^{3}$ Thus, digital health services can be especially beneficial for people in rural areas. ${ }^{15}$ 
Besides providing information and support services, digital platforms can improve the political and scientific participation of citizens, for example, by enabling them to express their individual needs via the online platform. Therefore, the aforementioned benefits of digitalisation for healthcare apply to research as well. Digitalisation facilitates broad participation of people eligible for studies, even for people living in rural areas. That applies to the identification and first contact with eligible participants as well as the conduction of online questionnaires in the studies itself.

\section{OBJECTIVES}

Deriving from the aforementioned challenges of dementia care and the benefits of digital technologies, the main objective of digiDEM is the improvement of the care situation of people with dementia and their family caregivers in the state of Bavaria. Bavaria is the largest German state by land area, comprising roughly a fifth of the total land area of Germany. With 13 million inhabitants, it is Germany's second-most-populous state after North Rhine-Westphalia. Therefore, the project is based on two pillars. One pillar is the set-up of a digital patient registry, including people with dementia and their family caregivers. In a longitudinal data collection process, digiDEM aims to examine the care situation in Bavaria in order to understand the long-term progression of dementia, the care situation in rural and urban areas, and the needs of people with dementia and their caregivers. This data are crucial for the future development and optimisation of national and regional structures in dementia care.

The second pillar of digiDEM aims to improve the care situation by establishing an online platform providing innovative digital services. This platform consists of four main types of digital services. First, digiDEM will provide digital psychosocial interventions for people with mild cognitive impairments (MCI) as well as people with mild and moderate dementia. One example is the evidencebased, structured MAKS (motorisch, alltagspraktisch, kognitiv, sozial) therapy. ${ }^{32}$ MAKS is a multicomponent psychosocial intervention, including exercises for social activation, sensorimotor activation, cognitive activation, and activation of activities of daily living (ADL). On average, MAKS therapy stabilised the outcomes 'cognitive abilities' and 'ADL abilities' over a period of 6 months, compared with a control group (usual care), in which these abilities deteriorated significantly. Effectiveness was proven in a randomised controlled trial (RCT) ${ }^{32}$ Participants will either get access to the manual and education for conducting the MAKS therapy in groups, or will be able to use the digital cognitive training MAKS-kog-ls. In a substudy of digiDEM about the identification of stakeholder's perspectives on the digital platform, results showed that particularly in regard to digital consulting and digital interventions, but also in regard to digital training, there is still a lack of digital services. ${ }^{33}$ MAKS-kog-ls is specifically developed for people with MCI and tailored to the individual therapeutic needs of each user. A review by Hill $e t a l^{34}$ showed that computerised cognitive training approaches have positive impacts on cognition in an early stage of the disease. Second, digiDEM focuses on the provision of support for caregivers. Third, digiDEM aims to support citizen volunteers in the care of people with dementia. In both cases, support will be provided interalia by education, for example, by means of videopodcasts and by facilitating communication in chats or forums. Recent reviews showed that psychoeducation is the most effective component of multicomponent interventions for caregivers and that it has positive effects on caregivers' wellbeing, quality of life, and mastery. ${ }^{35}{ }^{36}$ Lastly, the project wants to facilitate the participation of interested citizens. digiDEM will enable participation by surveying citizens' needs and preferences, asking for feedback for therapy and care services, or initiating contact between citizens and research projects. Based on these digital services, digiDEM aims to improve access to knowledge and structures in dementia care, in particular in rural areas. In combination with the digital psychosocial interventions, digiDEM can contribute to keep people with dementia in their home environment for a longer time and to support and disburden family caregivers.

The establishment of the digital patient registry is the first step in the project and will be explained in detail in the following.

\section{METHODS}

\section{Study design}

digiDEM is a multicentre, prospective, longitudinal register study that will be conducted in all administrative regions of Bavaria. The registry plans to recruit around 2000 participants who meet the inclusion criteria. The project is conceptualised, designed and initiated by a collaboration between the Friedrich-Alexander-University Erlangen-Nürnberg (FAU; represented by the Interdisciplinary Centre for Health Technology Assessment and Public Health and the Chair of Medical Informatics), the Universitätsklinikum Erlangen (represented by the Centre for Health Service Research) and the LeadingEdge Cluster Medical Valley EMN. digiDEM is part of the Bavarian Dementia Strategy (https://www.stmgp.bayern. de/pflege/demenz/), which was developed in 2013 by the Bavarian State Ministry of Health and Care. The project is conceptualised for fife years (2019-2023).

\section{Study population/participants}

Participants will be citizens with MCI and people with mild and moderate dementia. In order to identify potential participants, all people will undergo a screening based on the Mini-Mental State Examination (MMSE) ${ }^{37}$ and Montreal Cognitive Assessment (MoCA) ${ }^{38}$ screening tools prior to the inclusion process. Inclusion criteria for the people with dementia are an MMSE value between 15-23 points, living in their home environment, and 
primary residence in Bavaria. If the MMSE value is $>23$, the MoCA will be conducted additionally in order to identify people with MCI. If so, the range for inclusion will be 0-23 points. digiDEM will include all types of dementia and all age groups. People with an MMSE value between 0 and 14 points or a MoCA value $>23$ points, people who live in a nursing home, people with psychiatric diagnoses (depression, schizophrenia, addictions) or aphasia, and people that are deaf or blind will be excluded. People with advanced dementia will not be included in the study at baseline in order to ensure longer follow-up periods. If available, a caring relative will be included in conjunction with the person with dementia. Family caregivers will be included if they are the main caregiver of the respective person with dementia. As there is an increasing number of single households, digiDEM also wants to include people without a caregiver, in case the contact to the caregiver is less than one time per week. If there is a caregiver with frequent contact with the person with dementia, but the caregiver is not interested in participating in the project, there will be no inclusion of the person with dementia either. If participants reach an advanced stage of cognitive impairment and start receiving care from a family caregiver, the caregiver will be asked to participate in the project in order to ensure follow-up.

\section{Study recruitment}

digiDEM will recruit people with dementia and their family caregivers in all administrative regions of Bavaria. Participant recruitment will occur through research partners. Research partners are facilities that are embedded in the care of people with dementia and their family caregivers, such as community counselling, support groups, flat-sharing communities, memory clinics, day care hospitals, day care facilities, outpatient care organisations, acute care hospitals, district hospitals, geriatric rehabilitation facilities, and general practitioners.

\section{Patient and public involvement}

For the involvement of patients and stakeholders, eight workshops with family caregivers, professional caregivers, medical staff, and consultants for dementia care were performed in July and December 2019. Seven of those workshops were held with research partners from each of the seven administrative regions in Bavaria separately. The aim of the workshops was to lay the foundation for the cooperation with the research partners within the project. Therefore, research partners were asked to discuss their requirements for cooperation and to inform about regional specifics (for example, important players, demographic profiles, etc) that are relevant for the set-up of the registry infrastructure. One workshop, taking place in December 2019, was organised in order to develop a time-saving and valid research instrument for digiDEM in order to assess the need for and utilisation of support services of people with dementia and their family caregivers. Developing 'Dementia Assessment of Service Needs' (DEMAND) as a new assessment tool, participants were asked to identify existing support services for people with dementia and their caregivers as the fundament for the instrument and to discuss a first draft for the item design. Moreover, public involvement was ensured by an online survey in Bavaria from May to July 2019 in order to identify stakeholder's preferences for dementia research and their perspectives on the digital platform. Ninetyseven stakeholders participated in the survey. ${ }^{33}$ The information and feedback obtained from the workshops and the online survey were included in the project structure.

\section{Data protection}

digiDEM will comply with high standards of data protection. The project team developed a data protection concept according to the European General Data Protection Regulation. The concept was approved by the local Data-protection Supervisor of the FAU. Moreover, the data protection concept of digiDEM was authorised by the Bavarian Data Protection Commissioner. In order to ensure data protection, all personal data will be collected and stored separately from the registry data on different stand-alone systems. All participants will be pseudonymised. The collected data will be transferred to a secured server in encrypted form. Only authorised project staff will get access to the respective data.

\section{Data collection}

Data collection will take place over a period of three years (at baseline, after six months, 12 months, 24 months and 36 months). Data will be either collected by means of standardised online or face-to-face interviews using a web-based data entry system. Data collection via videoconference systems provides a secure option to do interviews even during the SARS-CoV-2 (COVID-19) crisis. The personnel of the research partners will be specifically trained by the digiDEM staff and afterwards conduct the interviews online or via direct contact in the home environment of the people with dementia. There will be frequent retraining for the research partners. The staff of the research partners will be responsible for the initial screening, explanation of the project, obtainment of informed consent and data collection. Both screening instruments MMSE and MoCA, are valid for audiovisual dementia screening. ${ }^{39} 40$ The project will collect sociodemographic data, data about the type of dementia, symptoms, diagnosis, cognitive trajectories (MMSE, MoCA), ${ }^{37} 38$ ADL (Barthel-Index), ${ }^{41}$ behavioural and psychological symptoms (Neuropsychiatric Inventory - NPI) ${ }^{42}$ falls, resource utilisation (Resource Utilization in Dementia - RUD) ${ }^{43}$ care situation, caregiver burden (Burden Scale for Family Caregivers - BSFC), ${ }^{44}$ social networks (Lubben Social Network Scale - LSNS), ${ }^{45}$ quality of life (EQ-5D, Short Form-12 - SF-12), ${ }^{46} 47$ needs of people with dementia and their caregivers (Dementia Assessment of Service Needs - DEMAND), mobility, use of media and sources of information. Making a dementia diagnosis is the responsibility of experienced clinicians, such as general practitioners, geriatricians, neurologists, 
and psychiatrists. In case the interview is performed in a memory clinic, the project will additionally gather information about comorbidities (Charlson-Index), ${ }^{48}$ risk factors, and diagnostic procedures (that is, imaging procedures and laboratory findings). Moreover, digiDEM will collect secondary data, like health and social data from the statutory health insurances, the Association of Statutory Health Insurance Physicians (Kassenärztliche Vereinigung), the pension insurance, and the Institute for Employment Research.

\section{Statistical analysis}

Descriptive statistics will be performed and presented in tables, including means, SD, and CIs. Univariate analyses and group comparisons will be conducted by means of $\mathrm{X}^{2}$ tests, $\mathrm{t}$-tests or Mann-Whitney $\mathrm{U}$ tests, and Pearson or Spearman correlation coefficients. Multivariate regression analysis will be used for examining associations between dependent and independent variables. Analysis of longitudinal data will be conducted by means of mixed analysis of variance (ANOVAs) and ANOVAs with repeated measures. A $p$ value $<0.05$ will be considered statistically significant.

\section{Technical aspects}

The project will implement a digital web-based platform for data collection, using Research Electronic Data Capture (REDCap) electronic data capture tools. ${ }^{49}{ }^{50}$ REDCap is a secure, web-based software platform designed to support data capture for research studies. REDCap enables the interviewers to capture data online via laptop and online or offline via tablet and smartphone. By doing so, data collection is also possible in places where no internet access is granted. REDCap is available at no charge to institutional partners. For online interviews, digiDEM will use the videoconference system Jitsi (https://jitsi.org/).

\section{Public relations}

digiDEM is engaged in public relations using several channels. digiDEM addresses citizens and potential research partners by participating in self-organised and external events, like the Bavarian Dementia Week. Moreover, the project appears on different social media channels. Furthermore, digiDEM provides specific knowledge sharing information (digiDEM Science Watch), Newsletters, and Webinars. digiDEM Science Watch informs the public about basic science, clinical research, dementia care research, and further up-to-date scientific developments and findings. The focus of the digiDEM Webinars is the education of professional and informal caregivers as well as citizens about dementia care, digital solutions, practical tools, and daily life recommendations.

\section{Ethics and dissemination}

digiDEM will be conducted in accordance with the provisions of the Declaration of Helsinki. The study obtained ethical approval from the Ethics Committee of the Medical Faculty of the FAU (application number: 253_20 B). Informed consent will be obtained from the participants or their authorised representative prior to screening and study inclusion. Informed consent will be obtained for screening, study inclusion, permission for data procession, and further contacting separately. Participation is voluntary.

Findings will be used for evidence-based decisionmaking for health decision-makers in order to optimise dementia healthcare in the state of Bavaria. Specific analyses will be conducted for the participating research partners. Study results will be published in national and international peer-reviewed journals and will be presented at national and international conferences. The journal articles will be available after publication at the homepage of the project. Moreover, digiDEM will prepare a final report after the data collection process is finished that will be published on the homepage of the Bavarian State Ministry of Health and Care.

\section{DISCUSSION}

The project digiDEM is characterised by some outstanding features. One specific element is the broad range of recruiting facilities. By recruiting people with dementia and their caregivers through community counselling, support groups, flat-sharing communities, memory clinics, day care hospitals, day care facilities, outpatient care organisations, acute care hospitals, district hospitals, geriatric rehabilitation facilities and general practitioners, digiDEM will include a heterogeneous patient population. Moreover, by focusing on rural areas, digiDEM will illuminate still neglected areas and contribute to filling the knowledge gap of dementia care in rural regions. A further outstanding aspect is that digiDEM will include people with dementia without age restrictions. Therefore, the project will be able to address people that are often not included in studies, as people with early-onset dementia. Another specific aspect is that digiDEM will link registry data to secondary data, which enables digiDEM to address multiple research issues, such as disease progression, needs and resource use, as well as health economic analyses. Finally, by providing innovative digital services, digiDEM additionally aims to improve the care situation of people with dementia and their family caregivers in Bavaria proactively. However, in this respect, both pillars of digiDEM have to be considered separately, as there is no direct link between the choice of the digital services and the data gathered in the research part of the project. The choice and implementation of digital services on the platform are based on former health outcomes research studies and studies proving the effectiveness of the interventions.

\section{Digital patient registries}

Patient registries are a crucial tool in health outcomes research. By observing clearly defined patient populations, registries can provide valuable data for, interalia, the utilisation, evaluation and future planning of services, the evaluation of treatment patterns or diagnostic 
information. ${ }^{51}$ In this regard, "[i]t is recommended that registries use web-based infrastructure, as user-friendly online technology facilitates data collection, processing, and reporting, ${ }^{52}$ Moreover, the use of digital technologies in patient registries provides some advantages, like the facilitation of plausibility tests in order to minimise the danger of missing or erroneous data during data collection or the reduction of errors during the data transmission process.

In a recent review about dementia registries worldwide, Krysinska et $a \tilde{l}^{2}$ indicated that digital tools are not yet widely used in the establishment of dementia registries. In many other areas of neurodegenerative diseases, however, digital registries are already successfully deployed and have proven to be valuable for many years. In multiple sclerosis (MS) research, the digital registry MSBase (https://www.msbase.org/) was developed in 2000. Meanwhile, MSBase is the largest international MS registry, including more than 63000 patient records from 132 member clinics in 35 countries ${ }^{53}$ Further digital MS registries were deployed in Sweden (SMSreg, 2001) and Norway (Multiple Sclerosis Registry and Biobank, 1998) ${ }^{5455}$ In the case of Huntington's disease, REGISTRY, a multilingual, multinational digital patient registry sponsored by the European Huntington's Disease Network (http://www.ehdn.org/), was set up in 2004, using an electronic web-based data capture system. ${ }^{56}$ In the USA, the National ALS Registry (https://www.cdc.gov/als/ Default.html) was launched in 2010. Within the registry, a secure web portal serves for data collection, allowing patients to self-enrol, provide demographic and diseaserelated information, and take brief risk factor surveys. ${ }^{57}$

\section{Digital dementia registries}

Although digital dementia registries not yet been widely established, they have been gaining more relevance recently in the course of digitalisation. In the USA, the Brain Health Registry (BHR) was set up in 2012. The aims of the US BHR are the collection of longitudinal data about dementia disease progression and fostering dementia research by means of research collaboration. The registry consists of a public website, registry, participant portal, investigator portal and database. Registration is conducted directly via the online platform. In order to register, people have to be at least 18 years old. After consenting to participate based on the information page, participants frequently undergo online self-report questionnaires and neuropsychological tests. Caregivers of the participants can register in a separate portal (Caregiver and Study Partner Portal) and, after consenting, complete questionnaires about the participant and the caregiver him/herself. Investigators can register as well, using the investigator portal for clinical trial recruitment. Thus, the Brain Health Project was able to reach 56982 consenting research partners until the beginning of $2018 .^{58}$ In collaboration with the US BHR, the Dutch BHR was developed and pretested in 2017. Derived from the US BHR, an online platform was developed, aiming to recruit, screen and monitor people potentially eligible for and interested in participating in dementia research. After registration, the research team initiates contact between eligible participants and dementia research projects, in particular clinical trials. ${ }^{59}$ Recently, the Australian Healthy Brain Project has been following a similar approach, using an online platform in order to recruit, assess and monitor Australian residents between 40 and 70 years of age. The central aim is to investigate longitudinal changes in cognition, lifestyle, mood and general health in order to identify people in the earliest stage of Alzheimer's disease and estimate the future risk of dementia. ${ }^{61}$ Further dementia projects using digital platforms are the Global Alzheimer's Platform Network, ${ }^{62}$ the Join Dementia Research (https://www.joindementiaresearch.nihr.ac.uk), and the European Prevention of Alzheimer's Disease. ${ }^{63}$

The facilitation of connecting participants and research studies is a huge benefit of patient registries in general and digital patient registries in particular. In this respect, the project digiDEM follows a broad approach by providing the possibility of bringing interested people in touch with other dementia studies, by doing research on its own, and by actively improving the care situation with digital services. In respect to the recruitment strategies of the aforementioned digital registries, the utilisation of an online registration and participation option may help to reduce participation barriers and therefore to reach many possible participants. However, lacking a direct contact, there is no option to either verify or validate the login details of the registered people or the results of self-report questionnaires, neuropsychological tests, and other relevant data. As digiDEM uses data in order to support political decision-making, data validity is a key issue. Thus, digiDEM is particularly focused on (online) face-to-face interviews with people with dementia and their caregivers.

\section{Digital services}

As aforementioned, digiDEM will provide digital services for people with dementia and their family caregivers. Digital services have the potential to support people with dementia and their family caregivers without geographical or time restrictions, which is particularly relevant for people living in rural areas. A study by Reichold et $a l^{33}$ showed that there is a demand for digital services in Bavaria. Nevertheless, the utilisation of digital tools for research or care is also accompanied by problems and barriers, in particular for the target group of digiDEM. Fang $e t a l^{64}$ showed that age is a particular factor in the existence of the digital divide. According to Friemel, the complexity of utilisation, the effort that has to be taken at the beginning as well as safety concerns, and technical problems are the main reasons of older adults for not using the internet. ${ }^{65}$

Author affiliations

${ }^{1}$ Interdisciplinary Center for Health Technology Assessment and Public Health (IZPH), Friedrich-Alexander University Erlangen-Nuremberg, Erlangen, Germany 
${ }^{2}$ Chair of Medical Informatics, Friedrich-Alexander University Erlangen-Nuremberg, Erlangen, Germany

${ }^{3}$ Medical Valley European Metropolitan Region Nuremberg Association, Erlangen, Germany

${ }^{4}$ Center for Health Services Research in Medicine, Department of Psychiatry and Psychotherapy, University Hospital Erlangen, Friedrich-Alexander University Erlangen-Nuremberg, Erlangen, Germany

Acknowledgements The present work was performed by Nikolas Dietzel in (partial) fulfilment of the requirements for obtaining the degree "Dr. rer. biol. hum." at the Faculty of Medicine of the Friedrich-Alexander University Erlangen-Nürnberg.

Contributors JT, H-UP, EG, and PLK-R initiated and planned the project. ND and PLK-R designed and conceptualised the study protocol and performed the literature review. ND wrote the study protocol. PLK-R supervised the protocol. ND, LKU, LKA, MR, SM, EG, and PLK-R developed the methodology. Technical implementation was done by MR, MH, MS, and H-UP. LKU, LKO, CC, SM, AK, KH, and MW performed quality control within the project. ND, LKU, MR, AN, MH, H-UP, EG, and PLK-R contributed to ethics and data protection. Patient and public involvement were carried out by ND, LKU, MR, CC, KS, MH, SM, AK, KH, MS, EG, and PLK-R. Critical revision of the protocol was done by MW, JT, H-UP, EG, and PLK-R.

Funding The project is funded by the Bavarian State Ministry of Health and Care as part of the funding initiative 'BAYERN DIGITAL II' (funding code: G42d-G8300-2017/1606-83).

\section{Competing interests None declared.}

Patient and public involvement Patients and/or the public were involved in the design, or conduct, or reporting, or dissemination plans of this research. Refer to the Methods section for further details.

Patient consent for publication Not required.

Provenance and peer review Not commissioned; externally peer reviewed.

Open access This is an open access article distributed in accordance with the Creative Commons Attribution Non Commercial (CC BY-NC 4.0) license, which permits others to distribute, remix, adapt, build upon this work non-commercially, and license their derivative works on different terms, provided the original work is properly cited, appropriate credit is given, any changes made indicated, and the use is non-commercial. See: http://creativecommons.org/licenses/by-nc/4.0/.

\section{ORCID iDs}

Nikolas Dietzel http://orcid.org/0000-0002-5820-3738

Peter L Kolominsky-Rabas http://orcid.org/0000-0002-7168-058X

\section{REFERENCES}

1 Ross J, Stevenson F, Lau R, et al. Factors that influence the implementation of e-health: a systematic review of systematic reviews (an update). Implement Sci 2016;11:146.

2 Labrique A, Vasudevan L, Mehl G, et al. Digital health and health systems of the future. Glob Health Sci Pract 2018;6:S1-4

3 World Health Organization. WHO guideline: recommendations on digital interventions for health system strengthening. World Health Organization, 2020. Available: URL: https://apps.who.int/iris/ bitstream/handle/10665/311941/9789241550505-eng.pdf?ua=1

4 European Commission. Communication from the Commission to the European Parliament, the Council, the European economic and social Committee and the Committee of the regions on enabling the digital transformation of health and care in the digital single market; empowering citizens and building a healthier Society. in Brussels 2018 https://ec.europa.eu/digital-single-market/en/news/ communication-enabling-digital-transformation-health-and-caredigital-single-market-empowering

5 European Commission. Commission staff working document accompanying the document communication from the Commission to the European Parliament, the Council, the European economic and social Committee and the Committee of the regions on enabling the digital transformation of health and care in the digital single market; empowering citizens and building a healthier Society. in Brussels 2018 https://ec.europa.eu/digital-single-market/en/news/ staff-working-document-enabling-digital-transformation-health-andcare-digital-single-market

6 World Health Organization. Dementia. A public health priority, 2018. Available: http://apps.who.int/iris/bitstream/handle/10665/75263/
9789241564458_eng.pdf;jsessionid=FOAFAE529500B048DC144018 11425F8F? sequence $=1$

7 Patterson C. World Alzheimer report 2018. The state of the art of dementia research: new frontiers, 2019. Available: https://www.alz. co.uk/research/WorldAlzheimerReport2018.pdf

8 Gustavsson A, Svensson M, Jacobi F, et al. Cost of disorders of the brain in Europe 2010. Eur Neuropsychopharmacol 2011;21:718-79.

9 Schaller S, Mauskopf J, Kriza C, et al. The main cost drivers in dementia: a systematic review. Int J Geriatr Psychiatry 2015;30:111-29.

10 Wimo A, Reed CC, Dodel R, et al. The GERAS Study: a prospective observational study of costs and resource use in community dwellers with Alzheimer's disease in three European countries--study design and baseline findings. J Alzheimers Dis 2013;36:385-99.

11 Kolominsky-Rabas PL, Gräßel E, Chilla T. Bayerischer Demenz survey (BayDem). Bericht für die Projektphase 2015-2018. Bayerisches Staatsministerium für Gesundheit und Pflege, 2020. Available: https://www.bestellen.bayern.de/application/eshop_ app000005?SID=1009396164\&ACTIONxSESSxSHOWPIC( BILDxKEY:\%27stmgp pflege_046\%27,BILDxCLASS:\%27Artikel\% 27,BILDXTYPE:\%27PDF\%27)

12 Speechly CM, Bridges-Webb C, Passmore E. The pathway to dementia diagnosis. Med J Aust 2008;189:487-9.

13 Chrisp TAC, Thomas BD, Goddard WA, et al. Dementia timeline: journeys, delays and decisions on the pathway to an early diagnosis. Dementia 2011;10:555-70.

14 Feldman L, Wilcock J, Thuné-Boyle I, et al. Explaining the effects of symptom attribution by carers on help-seeking for individuals living with dementia. Dementia 2017;16:375-87.

15 Barth J, Nickel F, Kolominsky-Rabas PL. Diagnosis of cognitive decline and dementia in rural areas - A scoping review. Int J Geriatr Psychiatry 2018;33:459-74.

16 Wimo A, Gauthier S, Prince M. Global estimates of informal care. Alzheimer's Disease International (ADI), 2020. Available: https://www. alz.co.uk/adi/pdf/global-estimates-of-informal-care.pdf

17 Cheng S-T. Dementia caregiver burden: a research update and critical analysis. Curr Psychiatry Rep 2017;19:64.

18 Karg N, Graessel E, Randzio O, et al. Dementia as a predictor of care-related quality of life in informal caregivers: a cross-sectional study to investigate differences in health-related outcomes between dementia and non-dementia caregivers. BMC Geriatr 2018;18:189.

19 McCabe M, You E, Tatangelo G. Hearing their voice: a systematic review of dementia family caregivers' needs. Gerontologist 2016;56:e70-88.

20 Brodaty H, Donkin M. Family caregivers of people with dementia. Dialogues Clin Neurosci 2009;11:217-28.

21 Lethin $\mathrm{C}$, Leino-Kilpi H, Roe B, et al. Formal support for informal caregivers to older persons with dementia through the course of the disease: an exploratory, cross-sectional study. BMC Geriatr 2016;16:32

22 Robinson KM, Buckwalter KC, Reed D. Predictors of use of services among dementia caregivers. West J Nurs Res 2005;27:126-40.

23 Phillipson L, Jones SC, Magee C. A review of the factors associated with the non-use of respite services by carers of people with dementia: implications for policy and practice. Health Soc Care Community 2014;22:1-12.

24 Karrer L, Dietzel N, Wolff F, et al. [Use of Outpatient Care Services by People with Dementia: Results of the Bavarian Dementia Survey (BayDem)]. Gesundheitswesen 2020;82:40-9.

25 Odzakovic E, Hydén L-C, Festin K, et al. People diagnosed with dementia in Sweden: what type of home care services and housing are they granted? A cross-sectional study. Scand J Public Health 2019;47:229-39.

26 Ruggiano N, Brown EL, Li J, et al. Rural dementia caregivers and technology: what is the evidence? Res Gerontol Nurs 2018;11:216-24.

27 Dzenowagis J. Digital technologies: shaping the future of primary health care World Health Organization, 2020. Available: https://www. who.int/docs/default-source/primary-health-care-conference/digitaltechnologies.pdf?sfvrsn=3efc47e0_2

28 Bhavnani SP, Narula J, Sengupta PP. Mobile technology and the digitization of healthcare. Eur Heart J 2016;37:1428-38.

29 Schaller S, Marinova-Schmidt V, Setzer M, et al. Usefulness of a tailored eHealth service for informal caregivers and professionals in the dementia treatment and care setting: the eHealthMonitor dementia portal. JMIR Res Protoc 2016;5:e47.

30 Herrmann LK, Welter E, Leverenz J, et al. A systematic review of Dementia-related stigma research: can we move the stigma dial? Am J Geriatr Psychiatry 2018;26:316-31.

31 Hehner S, Biesdorf S, Möller M. Digitalisierung Im Gesundheitswesen: die Chancen für Deutschland. New York: 
McKinsey \& Company, 2018. https://www.mckinsey.de/ / media/mckinsey/locations/europe\%20and\%20middle\%20east/ deutschland/news/presse/2018/2018-09-25-digitalisierung\%20im\% 20gesundheitswesen/langfassung $\% 20$ digitalisierung $\% 20 \mathrm{im} \%$ 20gesundheitswesen_neu.ashx

32 Straubmeier M, Behrndt E-M, Seidl H, et al. Non-pharmacological treatment in people with cognitive impairment. Dtsch Arztebl Int 2017; $114: 815-21$.

33 Reichold M, Dietzel N, Karrer L, et al. Stakeholder perspectives on the key components of a digital service platform supporting dementia - digiDEM Bayern. Stud Health Technol Inform 2020;271:224-31.

34 Hill NTM, Mowszowski L, Naismith SL, et al. Computerized cognitive training in older adults with mild cognitive impairment or dementia: a systematic review and meta-analysis. Am J Psychiatry 2017; 174:329-40.

35 Cheng S-T, Zhang F. A comprehensive meta-review of systematic reviews and meta-analyses on nonpharmacological interventions for informal dementia caregivers. BMC Geriatr 2020;20:137.

36 Parra-Vidales E, Soto-Pérez F, Perea-Bartolomé $\mathrm{M}^{\mathrm{a}}$ Victoria, Perea-Bartolomé MV, et al. Online interventions for caregivers of people with dementia: a systematic review. Actas Esp Psiquiatr 2017:45:116-26.

37 Folstein MF, Folstein SE, McHugh PR. "Mini-mental state". A practical method for grading the cognitive state of patients for the clinician. J Psychiatr Res 1975;12:189-98.

38 Nasreddine ZS, Phillips NA, Bédirian V, et al. The Montreal cognitive assessment, MoCA: a brief screening tool for mild cognitive impairment. J Am Geriatr Soc 2005;53:695-9.

39 Wadsworth HE, Galusha-Glasscock JM, Womack KB, et al. Remote neuropsychological assessment in rural American Indians with and without cognitive impairment. Arch Clin Neuropsychol 2016;31:420-5.

40 DeYoung N, Shenal BV. The reliability of the Montreal cognitive assessment using telehealth in a rural setting with veterans. $J$ Telemed Telecare 2019;25:197-203.

41 Mahoney FI, Barthel DW. Functional evaluation: the BARTHEL index. Md State Med J 1965;14:61-5.

42 Cummings JL, Mega M, Gray K, et al. The neuropsychiatric inventory: comprehensive assessment of psychopathology in dementia. Neurology 1994;44:2308-14.

43 Wimo A, Gustavsson A, Jönsson L, et al. Application of resource utilization in dementia (RUD) instrument in a global setting. Alzheimers Dement 2013;9:429-35.

44 Pendergrass A, Malnis C, Graf U, et al. Screening for caregivers at risk: extended validation of the short version of the burden scale for family caregivers (BSFC-s) with a valid classification system for caregivers caring for an older person at home. BMC Health Serv Res 2018;18:229.

45 Lubben J, Blozik E, Gillmann G, et al. Performance of an abbreviated version of the Lubben social network scale among three European community-dwelling older adult populations. Gerontologist 2006:46:503-13.

46 Orgeta V, Edwards RT, Hounsome B, et al. The use of the EQ-5D as a measure of health-related quality of life in people with dementia and their carers. Qual Life Res 2015;24:315-24.
47 Morfeld M, Kirchberger I, Bullinger M. SF-36 Fragebogen zum Gesundheitszustand: Deutsche version des short Form-36 health survey. Göttingen: Hogrefe, 2011.

48 Charlson ME, Pompei P, Ales KL, et al. A new method of classifying prognostic comorbidity in longitudinal studies: development and validation. J Chronic Dis 1987;40:373-83.

49 Harris PA, Taylor R, Thielke R, et al. Research electronic data capture (REDCap)--a metadata-driven methodology and workflow process for providing translational research informatics support. J Biomed Inform 2009;42:377-81.

50 Harris PA, Taylor R, Minor BL, et al. The REDCap Consortium: building an international community of software platform partners. $J$ Biomed Inform 2019;95:103208.

51 Richesson R, Vehik K. Patient registries: utility, validity and inference. Adv Exp Med Biol 2010;686:87-104.

52 Krysinska K, Sachdev PS, Breitner J, et al. Dementia registries around the globe and their applications: a systematic review. Alzheimers Dement 2017;13:1031-47.

53 Kalincik T, Butzkueven $\mathrm{H}$. The MSBase registry: informing clinical practice. Mult Scler 2019;25:1828-34.

54 Hillert J, Stawiarz L. The Swedish MS registry - clinical support tool and scientific resource. Acta Neurol Scand 2015;132:11-19.

55 Myhr K-M, Grytten N, Torkildsen Ø, et al. The Norwegian multiple sclerosis registry and Biobank. Acta Neurol Scand 2015;132:24-8.

56 Orth M, Handley OJ, Schwenke C, et al. Observing Huntington's disease: the European Huntington's disease network's registry. PLoS Curr 2010;2:Rrn1184.

57 Malek AM, Stickler DE, Antao VC, et al. The National ALS registry: a recruitment tool for research. Muscle Nerve 2014;50:830-4.

58 Weiner MW, Nosheny R, Camacho M, et al. The brain health registry: an Internet-based platform for recruitment, assessment, and longitudinal monitoring of participants for neuroscience studies. Alzheimers Dement 2018;14:1063-76.

59 Zwan MD, Flenniken D, Finley S, et al. [P2-052]: The dutch brain health registry: optimizing recruitment for dementia research. Alzheimer's \& Dementia 2017;13:P624.

60 Zwan MD, Flenniken D, Finley S, et al. P1-602: Dutch online Registry for recruitment of participants for dementia studies: Hersenonderzoek.NL and brain health registry. Alzheimer's \& Dementia 2018;14:P569-70.

61 Lim YY, Yassi N, Bransby L, et al. The healthy brain project: an online platform for the recruitment, assessment, and monitoring of middleaged adults at risk of developing Alzheimer's disease. J Alzheimers Dis 2019;68:1211-28.

62 Cummings J, Aisen P, Barton R, et al. Re-Engineering Alzheimer clinical trials: global Alzheimer's platform network. J Prev Alzheimers Dis 2016;3:114-20.

63 Ritchie CW, Molinuevo JL, Truyen L, et al. Development of interventions for the secondary prevention of Alzheimer's dementia: the European prevention of Alzheimer's dementia (EPAD) project. Lancet Psychiatry 2016;3:179-86.

64 Fang ML, Canham SL, Battersby L, et al. Exploring privilege in the digital divide: implications for theory, policy, and practice. Gerontologist 2019;59:e1-15

65 Friemel TN. The digital divide has grown old: determinants of a digital divide among seniors. New Media \& Society 2016;18:313-31. 